\title{
ELECTRONIC PROPERTIES OF InAs/GaAs SELF-ASSEMBLED QUANTUM DOT STRUCTURES AND DEVICES STUDIED BY PHOTOCURRENT SPECTROSCOPY
}

\author{
D.J. MoWbraY ${ }^{a *}$, P.W. FrY ${ }^{a}$, M.S. SKolNICK ${ }^{a}$, I.E. ITSKeVICH ${ }^{b}$, \\ L. HARris ${ }^{a}$, A.D. Ashmore ${ }^{a}$, J.J. Finley ${ }^{a}$, L.R. Wilson ${ }^{a}$, \\ K.L. Schumacher ${ }^{a}$, J.A. BarkeR ${ }^{c}$, E.P. O'Reilly ${ }^{c}$, M. Al-KhafaJi ${ }^{d}$,

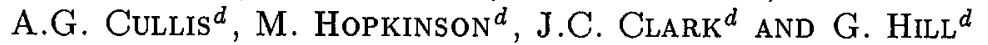 \\ ${ }^{a}$ Department of Physics and Astronomy, University of Sheffield, Sheffield S3 7RH, U.K. \\ ${ }^{b}$ School of Engineering, University of Hull, Cottingham Road, Hull HU6 7RX, U.K. \\ ${ }^{c}$ Department of Physics, University of Surrey, Guildford GU2 5XH, U.K. \\ ${ }^{d}$ Department of Electronic and Electrical Engineering, University of Sheffield \\ Sheffield S1 3JD, U.K.
}

The power of photocurrent spectroscopy to study the electronic properties of InAs/GaAs self-assembled quantum dots is described. From comparison of results from different samples it is shown that photocurrent provides a direct means to measure absorption spectra of quantum dots. Studies in high electric field enable the electron-hole vertical alignment to be determined. Most surprisingly this is found to be opposite to that predicted by all recent predictions. Comparison with theory shows that this can only be explained if the dots contain significant amounts of gallium, and have a severely truncated shape. The nature of the ground and excited state transitions, carrier escape mechanisms from dots, in-plane wave function anisotropies and the modal gain of a quantum dot laser are determined.

PACS numbers: 73.61.Ey, 78.66.Fd, 42.55.Px, 73.50.Pz

\section{Introduction}

Over recent years InAs/GaAs self-assembled quantum dots have been the subject of intense interest as a result of their discrete, zero-dimensional electronic states [1], their excellent opto-electronic quality and their favourable characteristics for novel applications such as ultra-low threshold lasers [1] and memory devices [2]. Despite this, many questions regarding their electronic properties remain unanswered due in part to the limitations inherent in many of the spectroscopic

*corresponding author: D.J. Mowbray, Department of Physics and Astronomy, University of Sheffield, Sheffield S3 7RH, UK. e-mail: D.Mowbray@Sheffield.ac.uk 
techniques employed. In particular absorption measurements which provide fundamental information (energies, oscillator strengths, polarisations, selection rules, etc.) on interband optical transitions and which are directly comparable to theoretical predictions, have been very difficult to perform. The only reports of absorption spectra have been from direct transmission measurements which required the use of extremely sensitive techniques with integration times of several hours per scan [3], and from the very low temperature technique of calorimetric absorption spectroscopy which appeared to yield broadened spectra [4].

In this work we demonstrate that photocurrent (PC) spectroscopy is a direct, sensitive and relatively simple technique to measure absorption spectra of quantum dots (the power of photocurrent spectroscopy for measuring absorption spectra of quantum wells has been pointed out by Collins et al. [5]). Studies in high electric field are shown to provide important information on the carrier wave functions in quantum dots (QDs), and by comparison with theory, on the composition, shape and effective height of the dots. We also determine the relative importance of different carrier escape mechanisms from the dots, the origin of the quantisation giving rise to the excited state transitions, perform polarised light spectroscopy and determine the modal gain of a quantum dot laser. We note that PC measurements at zero bias on InAs dots have been reported recently by Chu et al. [6] using an in-plane light propagation geometry. Qualitatively similar, but significantly less well-resolved spectra to those reported here, were obtained.

\section{Experimental}

All samples were fabricated using solid-source molecular beam epitaxy on GaAs (001) substrates. Both $p-i-n\left(p^{+}\right.$region uppermost) and $n-i-p\left(n^{+}\right.$region uppermost) were studied. For $p-i-n$ structures $250 \mathrm{~nm} n^{+}-\mathrm{GaAs}$ regions

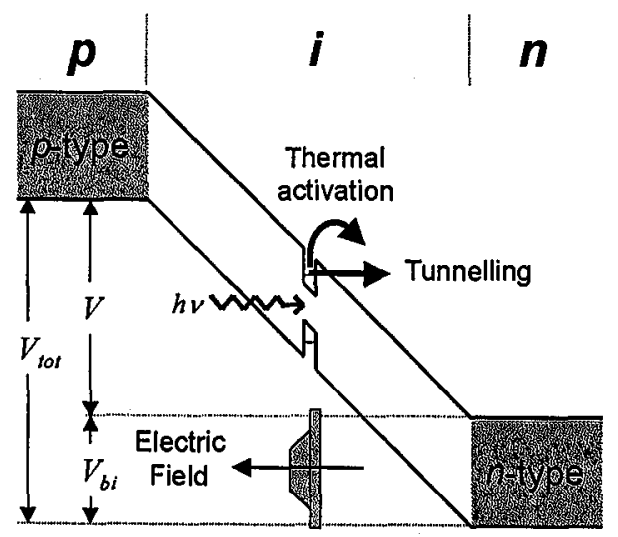

Fig. 1. Schematic band diagram under reverse bias of a $p-i-n$. The interband absorption process is indicated, followed by carrier escape either by tunnelling or by thermal excitation, thus leading to the observed photocurrent signals. The strongly truncated quantum dot shape employed to fit the observed Stark shift data is shown. 
were grown followed by a 300-550 nm undoped GaAs intrinsic region. 2.4 monolayer thick InAs quantum dot layers, grown by Stranski-Krastanov techniques at a temperature of $500^{\circ} \mathrm{C}$, were embedded in the centre of the intrinsic region. The intrinsic region was capped by a $300 \mathrm{~nm}$ thick $p^{+-}$GaAs contact layer. Reverse biasing results in an electric field oriented from substrate to surface. $n-i-p$ structures were fabricated by reversing the doping sequence, thus enabling electric fields to be applied in the opposite direction. The band profile for a typical $p-i-n$ device is illustrated in Fig. 1. For reverse applied bias, large fields could be applied with negligible dark current $(<1 \mathrm{nA})$ up to $\sim 10 \mathrm{~V}$, with the fields $(F)$ given by $F=\left(V+V_{\mathrm{bi}}\right) / W_{\mathrm{i}}$ where $V$ is the applied reverse bias, $V_{\mathrm{bi}}$ is the built-in potential $(\sim 1.5 \mathrm{~V})$ and $W_{\mathrm{i}}$ is the intrinsic region width.

Quantum dots grown under two distinct sets of growth conditions were investigated. The first type was grown at $\sim 0.01 \mathrm{ML} / \mathrm{s}$, leading to dots of $\sim 6 \mathrm{~nm}$ height and $\sim 18 \mathrm{~nm}$ base length as evidenced by cross-sectional transmission electron microscopy (TEM) as shown in Fig. 2a. From plan-view TEM the areal density was found to be $3.5 \times 10^{10} \mathrm{~cm}^{-2}$. For the second dot type the InAs was deposited
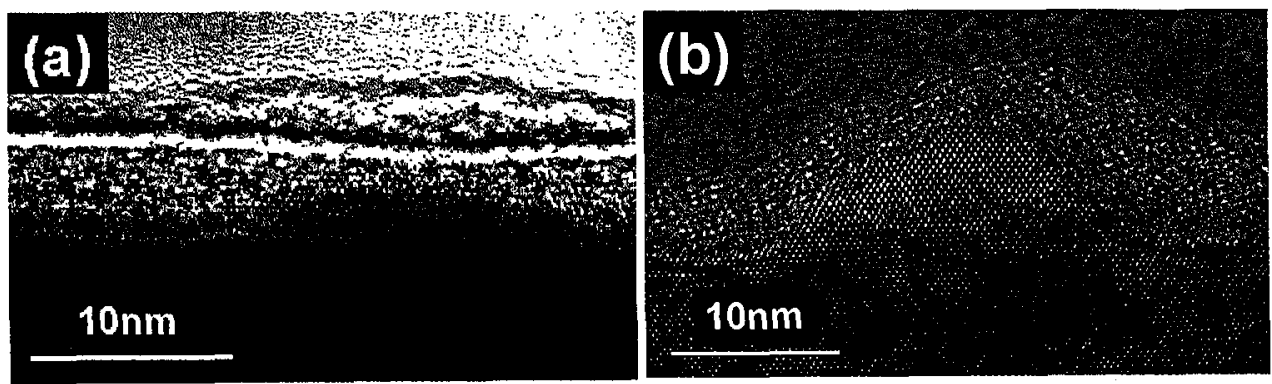

Fig. 2. Cross-sectional transmission electron micrographs of uncapped slow (a) and fast (b) growth rate quantum dots.

TABLE

Details of sample structures.

\begin{tabular}{c|c|c|c|c}
\hline \hline $\begin{array}{c}\text { Sample } \\
\text { number }\end{array}$ & Device type & $\begin{array}{c}\text { Total intrinsic } \\
\text { width, } W_{\mathrm{i}}[\mathrm{nm}]\end{array}$ & $\begin{array}{c}\text { InAs deposition } \\
\text { rate [ML/s] }\end{array}$ & $\begin{array}{c}\text { No. of dot } \\
\text { layers/spacer } \\
\text { width [nm] }\end{array}$ \\
\hline 1 & $p-i-n$ & 500 & 0.09 & 1 \\
2 & $n-i-p$ & 525 & 0.087 & 1 \\
3 & $n-i-p$ & 550 & 0.087 & $5 / 25$ \\
4 & $p-i-n$ & 300 & 0.01 & 1 \\
5 & $p-i-n$ & 300 & 0.01 & 1 \\
6 & $n-i-p$ & 300 & 0.01 & 1 \\
7 & $p-i-n$ & 550 & 0.09 & 1
\end{tabular}


at the faster rate of $0.09 \mathrm{ML} / \mathrm{s}$. This produced smaller dots with an approximate height of $3 \mathrm{~nm}$ (base $\sim 15 \mathrm{~nm}$ ) as shown in Fig. $2 \mathrm{~b}$, and higher dot density of $9.6 \times 10^{10} \mathrm{~cm}^{-2}$.

The samples studied are summarised in the Table. Samples 2, 3 and 7 were designed as laser structures and contain additional $1.5 \mu \mathrm{m}$ thick $\mathrm{Al}_{0.6} \mathrm{Ga}_{0.4} \mathrm{As}$ cladding layers either side of the GaAs intrinsic region. Photocurrent spectra were investigated using lock-in techniques with the samples illuminated by monochromated white light from a tungsten halogen lamp. Low intensity jllumination of $\sim 3 \mathrm{~mW} \mathrm{~cm}{ }^{-2}$ was employed, corresponding to dot occupancies $\ll 1$ where the spectra are unperturbed by many carrier occupation effects.

\section{Results and discussion}

\subsection{General aspects of photocurrent spectra}

In this section we present PC spectra at zero applied bias, and investigate how the spectra depend on areal density and number of dot layers. In Fig. 3a, we show PC spectra for samples 1 (S1 fast growth) and 4 (S4 slow growth) at a temperature of $300 \mathrm{~K}$. An electroluminescence (EL) spectrum of sample S4, obtained by forward biasing the device, is shown for comparison. The PC spectra consist of three (S1) or four (S4) features arising from interband transitions in the QDs, superimposed on a broad background signal which strongly increases in intensity to higher energy. As indicated schematically in Fig. 1, electron-hole pairs created by interband absorption escape from the dots and give rise to the measured photocurrent. The carrier escape mechanisms are analysed in Sec. 3.4. However for the moment, the important point is that for zero applied bias at temperatures $>200 \mathrm{~K}$ (the present experimental conditions) all carriers created by interband absorption escape from the dots and contribute to the photocurrent. As a result it is expected that the PC spectra provide a quantitative representation of the absorption spectra of the QDs.

This expectation is supported by the results in Figs. 3a and b. In Fig. 3a the magnitude of the PC signal for the fast growth dots (S1) is found to be $\sim 3$ times greater than for the slow growth dots (S4), consistent with the $\sim 3$ times higher density of these dots. In Fig. 3b, we compare spectra from samples S2 and S3, fast growth dots containing one and five dot layers, respectively. The five dot layer sample was grown with spacer layers of $25 \mathrm{~nm}$, thus ensuring that there is no electronic coupling between the successive layers. The PC spectra of the two samples are very similar in overall form to those in Fig. 3a. Most importantly the intensities of the transitions in S3 are, to a very good approximation, a factor of five stronger than those in S2, as seen from the close similarity in intensity between the S3 spectrum and the dotted curve corresponding to the S2 spectrum multiplied by a factor of five. The results in Figs. 3a and $b$ thus both demonstrate that the PC signals are directly proportional to the number of dots in the samples and thus suggest strongly that true absorption spectra are being measured.

As well as permitting the form of the absorption spectra of the QDs to be deduced, $\mathrm{PC}$ also enables absolute values for the absorption strength $(A)$ to be obtained. Under conditions where all the photocreated carriers escape from the QDs, 

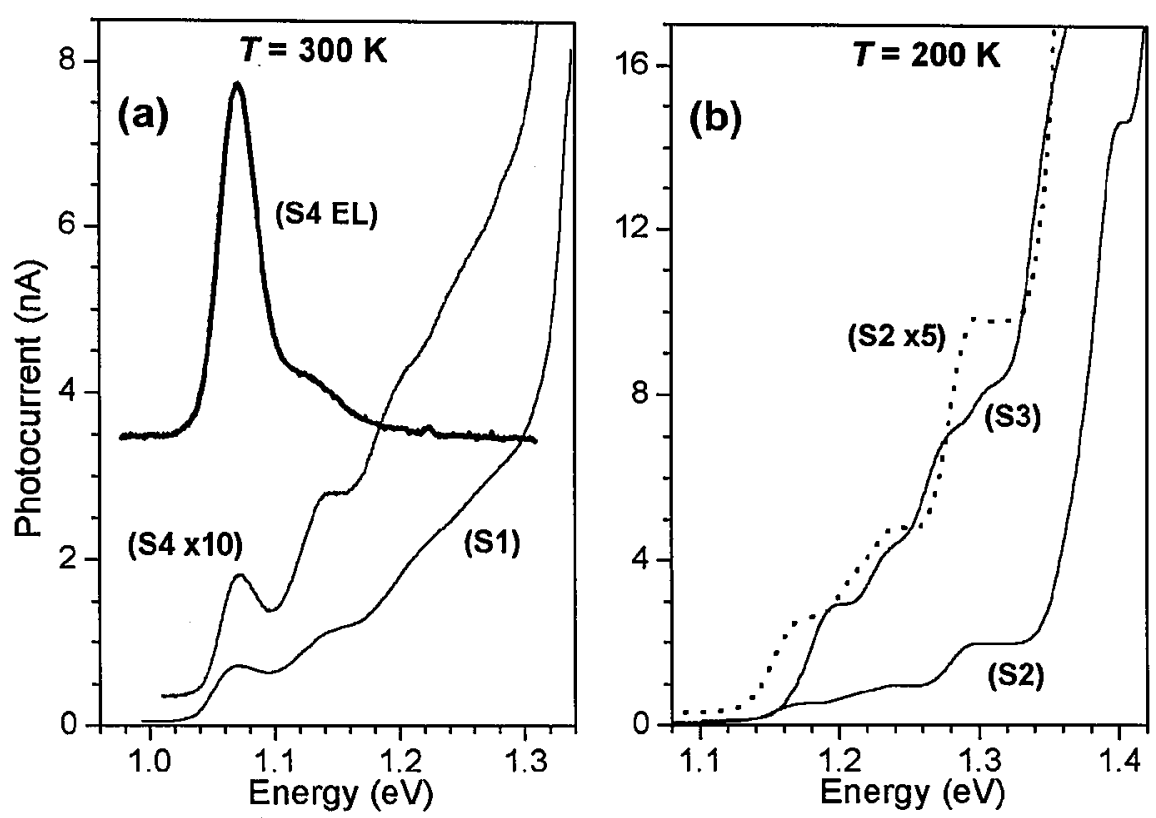

Fig. 3. (a) Photocurrent spectra at $300 \mathrm{~K}$ and zero applied bias for samples S1 (slow growth) and S4 (fast growth) showing 3 (S1) and four (S4) interband transitions. Also shown is an electroluminescence spectrum of sample $\mathrm{S} 4$ for comparison with the photocurrent spectrum. (b) Comparison of single layer (S2) and 5 layer (S3) dot samples.

and hence contribute to the PC signal, the magnitude of the photocurrent signal $I$ is related to the total incident optical power $P$ at frequency $\nu$ by $I=A P e / h \nu$. To determine $I$ for the ground state transition a (small) linear background subtraction was performed. Using this procedure we obtain peak values of $A$ for the ground state transitions of $(2.8 \pm 0.3) \times 10^{-4}$ for sample 1 and $(8.7 \pm 1.2) \times 10^{-5}$ for sample 5 . These values are in good agreement with that reported by Warburton et al. from direct transmission spectroscopy [3] after taking into account the different areal dot densities. It is also interesting to compare the relative size of the photocurrent signals from the QDs and from the wetting layer (representative of a two-dimensional quantum well) signal at $1.38 \mathrm{eV}$. For sample $\mathrm{S} 1$ the integrated area of the dot ground state signal is found to be $0.06(1 / 17)$ of that of the wetting layer. This value agrees reasonably well with the fractional areal coverage of the dots in this sample of 0.1 , thus showing that any enhancement of exciton oscillator strength due to the extra confinement in dots relative to quantum wells is small.

As noted in the introduction, the absorption measurements of Ref. [3] required very long measurement times and a highly stable experimental system. By comparison, the absorption spectra measured by PC are obtained by very straightforward experimental techniques in typical measurement scans of 5 to 10 minutes. The main reason for the high sensitivity of the $\mathrm{PC}$ techniques is that $\mathrm{PC}$, unlike direct absorption, is a zero background technique and furthermore currents can very easily be measured with sub $\mathrm{pA}$ sensitivity. 


\subsection{Electric field dependence of spectra}

Spectra for sample 5 are shown in Fig. 4 as a function of field at a temperature of $200 \mathrm{~K}$, with the variation of the ground state transition energies plotted in the inset, together with those from sample 6 , the $n-i-p$ complement of sample 5 . A strong shift of the interband transitions to lower energy is observed, due to the quantum confined Stark effect. In addition the transition energies exhibit a marked asymmetry about $F=0$, with the maximum obtained for a non-zero field of $-90 \mathrm{kV} / \mathrm{cm}$. The asymmetry implies that the QDs have a permanent dipole moment $(p)$. The field dependence of the transition energies $(E)$ in Fig. 4 can be described to a very reasonable approximation by the expression $E=E_{0}+p F+\beta F^{2}$ (see Ref. [7]) where $E_{0}$ is the energy at $F=0$, the second term arises from the non-zero dipole moment $(p)$, and the third term from polarisation of the dots in the applied field (the quantum confined Stark effect). By fitting to the experimental data for samples 4 and 5 a value of $p=(7 \pm 2) \times 10^{-29} \mathrm{Cm}$ is determined, corresponding to an electron-hole separation of $r=4.0 \pm 1 \AA$ (from $p=e r$ ).

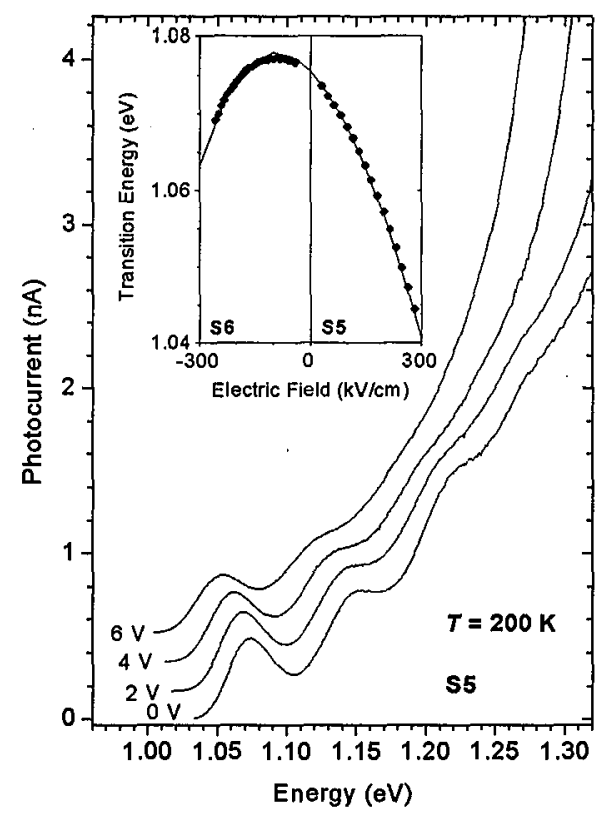

Fig. 4. Photocurrent spectra for sample S5 as a function of applied bias at a temperature of $200 \mathrm{~K}$. A strong Stark shift to lower energy of the interband transitions is seen. The inset shows the ground state transition energies for the consecutively grown $p-i-n$ and $n-i-p$ samples (samples S5 and S6) as a function of electric field, together with a theoretical fit.

Self-assembled QDs are expected to have a permanent dipole moment due to their asymmetric shape [8]. However, the experimentally determined direction of the dipole is very surprising. The maximum transition energy occurs for $F$ in 
the direction from apex to base, corresponding to electron (hole) attraction to the apex (base) of the dots. This implies that the electron charge density distribution lies below that of the hole at $F=0$, with the dipole pointing from base to apex. This result, to the best of our knowledge, is in contrast to that predicted by all accurate modelling [8-10] which predicts hole localisation towards the base of the dots, below the electrons. This alignment, which occurs for pure InAs dots of any shape for which the lateral size decreases from base to apex (e.g. the pyramidal shape in Refs. [8-10] results from the strain-induced form of the valence band edge profile $^{\dagger}$, and the difference in the effective mass ratios along the growth direction $\left(m_{\mathrm{hh}}^{*} \gg m_{\mathrm{e}}^{*}\right)$.

To determine dot parameters necessary to reverse the alignment, and to fit the experimental data, envelope function calculations, treating the electrons and holes with separate one-band Hamiltonians, have been performed. As a result of the calculations, further details of which can be found in Refs. [7, 11], we are able to deduce that the inverted electron-hole alignment is only possible for dots having a graded $\mathrm{In}_{1-x} \mathrm{Ga}_{x}$ As composition, with $x$ decreasing from base to apex. In addition it is also necessary to severely truncate the pyramid to achieve a dipole of the correct sign, since strain effects localise the hole strongly below the electron until the truncation factor is greater than $\approx 0.6$. The best fit to the data is shown by the full line in the Fig. 4 inset. This was obtained using a pyramid of base length $(w) 15.5 \mathrm{~nm}$, height $22 \mathrm{~nm}$, of which the top $75 \%$ is truncated (see Fig. 1) to give an actual height $(d)$ of $5.5 \mathrm{~nm}$, and an $x$ varying linearly from 0.5 at the base to zero at the top surface. Although other parameter sets can give a good fit, we emphasise that the shape employed represents a good approximation to that obtained from structural measurements (Fig. 2). Furthermore full pyramids (or any shape wider at the base than the apex) of constant composition, or full pyramids with linear grading, never have a dipole of the correct sign.

It is interesting to note that all the interband transitions of Fig. 4 show the same shift with applied field. This shows that the excited state features must arise from transitions between laterally confined states, since if they were due to vertical quantisation, as in quantum wells, they would show Stark shifts different from that of the ground state.

\subsection{Polarisation dependent spectra}

We have shown in Sec. 3.1 that PC represents a direct means to measure the absorption spectra of QDs, and have discussed an application of $\mathrm{PC}$ techniques to investigate the effects of applied electric fields. We now show that PC can be used to investigate polarisation dependent properties.

\subsubsection{In-plane propagation}

We first present spectra for light propagating in the growth plane of the QDs. These results were obtained on samples S2 and S7, samples grown with $\mathrm{Al}_{0.6} \mathrm{Ga}_{0.4} \mathrm{As}$ cladding layers, required to achieve efficient in-plane light guiding. Spectra are presented in Fig. 5 for TE (electric vector in the plane of the QDs) and TM (electric vector parallel to the growth direction) polarisations. For both

\footnotetext{
tThe strain acts to localise the holes in the widest region of the dots.
} 


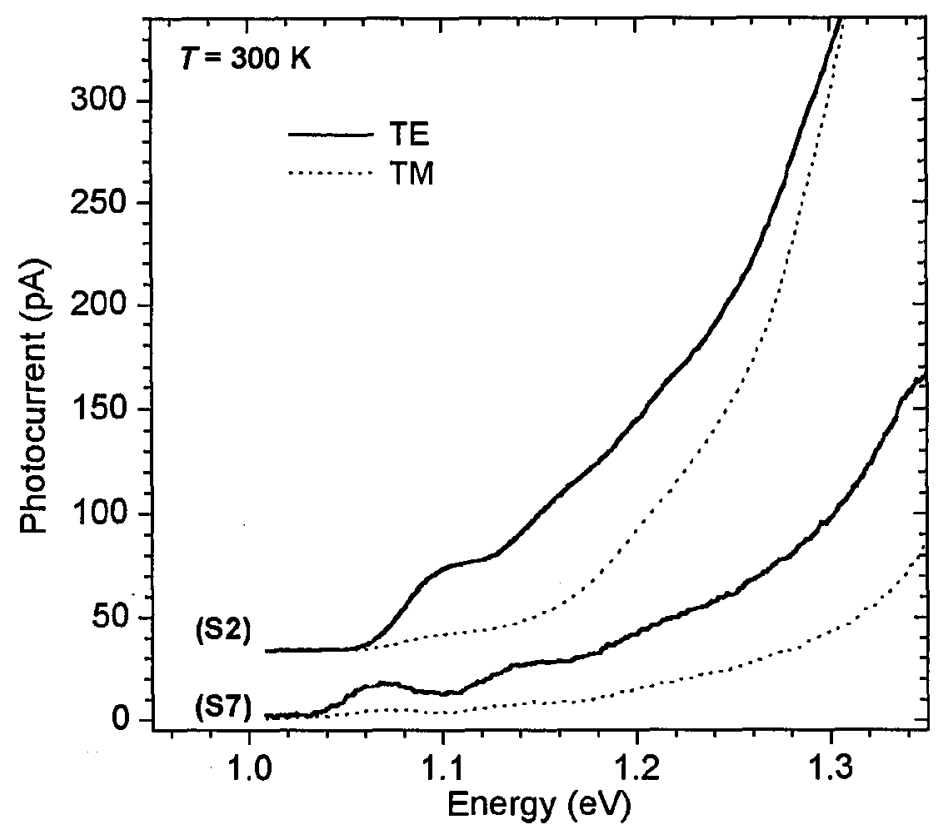

Fig. 5. Polarisation dependent spectra for in-plane propagation for samples S2 and S7. The spectra are observed to be strongly TE polarised (electric vector in the plane of the layers), as expected for transitions involving predominantly heavy-hole-like valence band states.

samples the spectra are observed to be strongly TE polarised (especially for S7 where the TE spectra are a factor of 6 stronger). As mentioned briefly in Ref. [7], and also in Ref. [6], this shows that the lowest energy states are predominantly heavy hole in character [12], an important assumption of the theoretical modelling of Refs. [7] and [11].

\subsubsection{Normal incidence}

There have been several theoretical predictions that QD transitions should exhibit anisotropy within the growth plane of the dots, arising from the presence of piezoelectric fields in the structure [13], or from the microscopic inequivalence of the $(110)$ and $(-110)$ directions [14]. To examine whether such anisotropies could be detected experimentally we measured normal incidence PC spectra for sample S1, for light polarised parallel to different crystallographic directions in the growth plane. In the spectral region of the dot transitions little polarisation dependence is found, with orthogonal polarisations agreeing within $5 \%$. Spectra for the higher energy region extending beyond the GaAs band gap exhibit no detectable polarisation, as expected, thus showing the polarisation independence of the measurement system.

The observation of at most very weak polarisation of the quantum dot PC spectra, with in addition no significant differences between ground and excited 
state transitions, agrees only moderately with the predictions of Refs. [13] or [14] for faceted pyramidal dots where polarisations in excess of $25 \%$, especially for the excited states are predicted. The reasons for this disagreement are not fully clear, but may include variation of the degree of polarisation between different dots due to shape variations, or possibly to some omissions in the theories (it should be noted that Ref. [14] predicts smaller polarisations for dots with circular base). We also note that polarisations of $\sim 20$ and $40 \%$ have been reported in PL spectra under high power [15]. For the moment it is thus difficult to draw definitive conclusions from the in-plane polarisation measurements. Measurements of polarisation ratios on single dots in the same sample are likely to be very helpful in clarifying the situation, since they would eliminate the possibility of polarisation averaging effects.

\subsection{Carrier escape mechanisms}

We now turn attention to analysis of the carrier escape mechanisms. As indicated schematically in Fig. 1, electron-hole pairs created by interband absorption escape from the quantum dots and are swept to the contacts to give rise to the observed photocurrent. In order to understand the nature of the escape mechanisms, photocurrent spectra were recorded over a wide range of temperature and electric field.

Figure 6 a displays a series of spectra from sample 4 under zero applied bias (electric field of $50 \mathrm{kV} / \mathrm{cm}$ ) for temperatures from $5 \mathrm{~K}$ to $200 \mathrm{~K}$. At $5 \mathrm{~K}$ there are no observable interband dot features, a weak signal being observed only at energies' $>1.25 \mathrm{eV}$, from band-tail absorption of the wetting layer and GaAs cladding regions, as discussed previously. As the temperature is raised to $\sim 120 \mathrm{~K}$, well-defined but weak features appear in the spectrum. In the range of 130-200 K the peaks become progressively more intense with increasing temperature, reflecting the growing probability for carrier escape as the thermal energy increases; for these low applied fields, carrier escape from the dots by thermal activation is thus only significant for temperatures $>\sim 120 \mathrm{~K}$.

It is notable that the PC spectra for the ground and excited state transitions all show the same temperature dependence. This indicates that following interband excitation, thermally activated escape always occurs from the same confined energy levels (very likely the electron/hole ground states), and thus that the relaxation rate from excited to ground states $\left(\sim 3 \times 10^{11} \mathrm{~s}^{-1}\right)[16]$ is faster than any competing escape rate. This observation provides strong evidence that the excited states arise from lateral quantisation ${ }^{\ddagger}$, since if they were due to vertical quantisation, tunnel escape would be expected to be dominant. Using Wentzel-Kramers-Brillouin (WKB) methods, we estimate very rapid tunnelling rates of $>10^{12} \mathrm{~s}^{-1}$ for an

\footnotetext{
\The dominant quantisation arises in the vertical direction along which the dots have their smallest dimension. This quantisation provides the major contribution to the ground state energy of the dots (energy upshifts by $>200 \mathrm{meV}$ ). The excited states arise from additional lateral quantisation (lateral $p$ and $d$ states in harmonic oscillator notation) with typical quantisation energies of $70 \mathrm{meV}$ (electron plus hole). The results presented in the present paper for both the carrier escape and the Stark effect of the excited states in Sec. 3.1 show that a decoupled approximation to treat the vertical and lateral dot quantisation is a good one.
} 
excited state of ionisation energy of $50 \mathrm{meV}$ even for electric fields of less than $50 \mathrm{kV} / \mathrm{cm}^{\S}$. This conclusion regarding the lateral quantisation origin of the excited states is consistent with that obtained in Sec. 3.1 from the observation of identical Stark shifts for all the QD interband transitions.
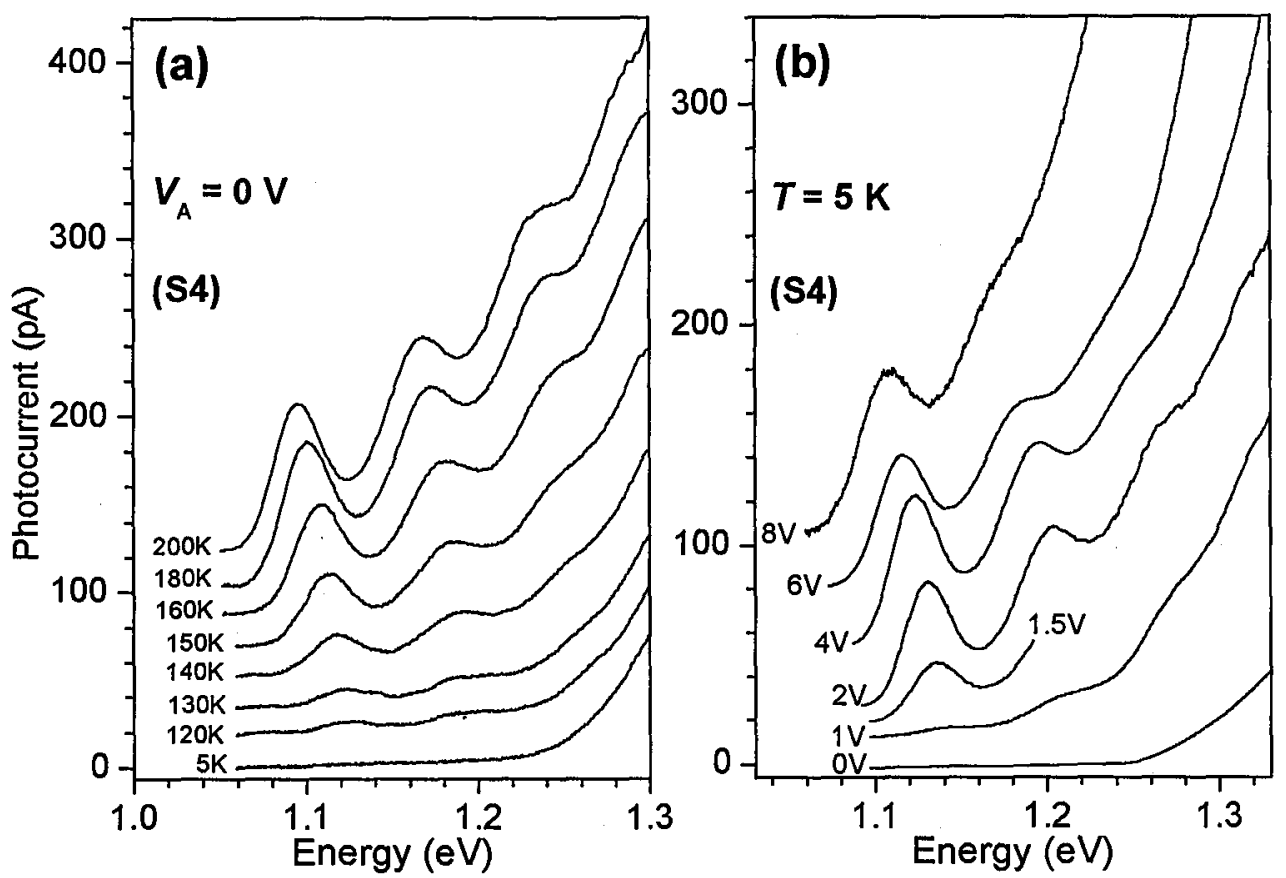

Fig. 6. (a) Zero bias spectra for sample $\mathrm{S} 4$ for temperatures from 5 to $200 \mathrm{~K}$. At the low bias of $0 \mathrm{~V}$ and for temperatures less than $\sim 100 \mathrm{~K}$, only very weak signals are observed from the interband QD transitions. For higher temperatures all the interband transitions show the same strongly thermally activated behaviour. (b) Spectra at $5 \mathrm{~K}$ as a function of reverse bias. The interband QD transitions, only have significant strength for reverse biases in excess of $\sim 1.5 \mathrm{~V}$, showing the dominance of tunneling escape at low temperature.

In Fig. 6b spectra are presented as a function of bias $(V)$, at a low temperature of $5 \mathrm{~K}$ where there is no escape by thermal excitation. As in Fig. 6a $(T<100 \mathrm{~K})$ at low bias $(<0.5 \mathrm{~V}$, electric field $<65 \mathrm{kV} / \mathrm{cm})$, only the band tail signal from the wetting layer/GaAs is observed. With increasing bias, the well-defined interband transitions become increasingly prominent and reach their full intensity for biases greater than $3 \mathrm{~V}$ (fields $>150 \mathrm{kV} / \mathrm{cm}$ ) when all carriers escape from the dots. This behaviour is consistent with tunnelling being the dom-

\footnotetext{
$\S$ For this estimate we approximated the dot by a $50 \AA$ wide potential well, assumed an electron mass of $0.05 m_{e}$ and took the energy separation $\left(\Delta E_{s}\right)$ between the excited state and the continuum to be $50 \mathrm{meV}$.
} 
inant carrier escape mechanism at low temperature, with escape again occurring from a common level, since all the interband transitions show the same bias dependence. The PC signal is expected to reach its full intensity with increasing field when the tunnelling time of the carriers which can tunnel most rapidly from the dots becomes less than the radiative recombination time $(\sim 1 \mathrm{~ns})$ [17]. WKB estimates of tunnelling times show that for a field of $\sim 100 \mathrm{kV} / \mathrm{cm}$ when tunnelling is first significant, ground state tunnelling times are expected to be in the range 1 to $10 \mathrm{~ns}$ for electrons, and many orders of magnitude greater for heavy holes due to the $>5$ times higher hole mass $\pi$. Such estimates (probably only accurate to within an order of magnitude) are well known to be highly sensitive to input parameters (carrier masses, band offsets, etc.). They nevertheless are consistent with the conclusion that the photocurrent signal becomes observable at low temperature when electron tunnelling from the wells occurs.

Thus to summarise this section, we have shown that at low temperature, escape is dominated by tunnelling, whereas with increasing temperature, thermal escape becomes increasingly important and dominates for temperatures in excess of $\sim 200 \mathrm{~K}$. In both cases it has been seen that escape occurs from a common level in the dots, probably the electron/hole ground states.

\subsection{Quantum dot lasers}

There is considerable interest in the use of quantum dots as the active region of injection lasers due to theoretical predictions of the resultant low threshold current density, temperature independent threshold current density and large differential gain [1]. However, although impressive performances have been reported for self-assembled QD lasers [19] there is considerable evidence that the basic model gain is relatively small [20], probably as a result of the low fractional area occupancy of the dots. In this section we show how PC measurements can be used to determine the modal gain of a quantum dot laser and investigate the consequences for device performance of the low value found.

$\mathrm{PC}$ measurements of the laser structure S7, which contains a single layer of dots, give a value of $A=(2 \pm 0.6) \times 10^{-4}$ for the fractional plane wave absorption for normal incidence. To determine the modal gain $(g)$ for inplane propagation of light in a wave guide structure, the corresponding in-plane absorption coefficient $(\alpha(\equiv-g))$ is calculated. This is obtained in two steps. First, an effective plane wave absorption coefficient is determined by considering a sequence of parallel dot planes, with adjacent planes separated by the average dot spacing $D$. Second, this absorption coefficient, which is equivalent to the plane wave absorption in an infinitely wide planar cavity containing an infinite number of dot planes, is multiplied by a geometrical factor $\left(\Gamma_{\mathrm{ef}}\right)$ to convert to the wave guide geometry consistent with a guided mode and a single dot plane. $\alpha$ is therefore given by

I As for the excited state estimate in the footnote $§$ (see previous page), tunnelling times were calculated for a $50 \AA$ InAs qantum well in GaAs barriers, assuming a 60:40 split in the offset between the conduction band and valence band, with $m_{\mathrm{e}}=0.05 m_{\mathrm{e}}, m_{\mathrm{hh}}=0.35 m_{\mathrm{e}}$. Similar conclusions regarding the respective electron and hole tunnelling times are reached in Refs. [2] and $[18]$. 
$\alpha=\ln \left((1-A)^{1 / D}\right) \Gamma_{\mathrm{ef}} ;$ where the first term $\left(\ln \left((1-A)^{1 / D}\right) \approx A / D\right.$ as $\left.A \ll 1\right)$ is the plane wave absorption coefficient. $\Gamma_{\mathrm{ef}} \|$ is calculated for the present device using a standard symmetric three-layer (two $\mathrm{Al}_{0.6} \mathrm{Ga}_{0.4}$ As cladding layers and a GaAs cavity) slab wave guide model [21]. For the present structure a value $\alpha=$ $(7 \pm 3) \mathrm{cm}^{-1}$ is obtained for the ground state modal absorption. Because the maximum gain for a $Q D$ transition has a numerical value equal to $\alpha$ [22], the maximum ground state modal gain, $g_{\bmod }^{\max }$, for the present single layer device is $(7 \pm 3) \mathrm{cm}^{-1}$. Although the light propagation direction for the the photocurrent measurements is normal to that for inplane wave guide propagation, the inplane polarised PC results of Sec. 3.3 demonstrate that the ground state emission has an inplane (TE) polarisation. Hence the polarisation direction for the two configurations is identical.

The $(7 \pm 3) \mathrm{cm}^{-1}$ value for $g_{\bmod }^{\max }$ is comparable to values $3.5 \sim 8 \mathrm{~cm}^{-1}[20,23]$, deduced for similar devices from a determination of the threshold modal gain as a function of cavity length. These values of $g_{\text {mod }}^{\max }$ are significantly smaller than those of comparable quantum well (QW) lasers, where values of $\sim 50-100 \mathrm{~cm}^{-1}$ are typical for InGaAs-based devices [21, 24]. Although QDs are predicted to exhibit a higher material gain than QW lasers [25] their modal gain may be considerably smaller as a result of a relatively small effective transverse optical confinement factor $\Gamma=\Gamma_{z} f$, where $\Gamma_{z}$ is the overlap of the dots and optical field along the growth direction and $f$ is the fractional area occupancy of the dots. For the present structure $\Gamma_{z}=0.015$ and $f=0.1$, giving $\Gamma=1.7 \times 10^{-3}$. In contrast, for a QW laser $\Gamma_{z}$ will generally be larger, due to the greater $\mathrm{QW}$ thickness along the growth direction (typically $\sim 8-10 \mathrm{~nm}$ compared to an effective value of $2 \mathrm{~nm}$ for the QDs), and $f$ has a value of unity. In the light of these considerations the relative values of $g_{\bmod }^{\max }$ for a QD and QW laser are reasonable.

The small value determined for $g_{\bmod }^{\max }$ is comparable to the internal cavity loss $\left(\alpha_{i}\right)$, which typically has a value in the range of $\sim 2-10 \mathrm{~cm}^{-1}[20]$, and thus lasing on the ground state transition may be difficult to achieve. Such lasing is desirable, as carriers in the corresponding dot states are the most strongly confined, hence minimising thermal carrier loss from the dots and resulting in the optimum temperature performance. Figure 7 shows emission spectra for a $2 \mathrm{~mm}$ cavity laser device fabricated from the $\mathrm{S} 7$ wafer. The emission from the ground state transition saturates at low currents, indicating that the gain of this transition is insufficient to overcome $\alpha_{i}$ plus the mirror losses $\left((1 / L) \ln (R) \approx 5.7 \mathrm{~cm}^{-1}\right)$. A similar behaviour is observed for the first excited state transition and lasing eventually occurs via the second excited state transition. A $5 \mathrm{~mm}$ cavity device exhibits a similar behaviour except that the reduced mirror loss $\left((1 / L) \ln (R) \approx 2.3 \mathrm{~cm}^{-1}\right)$ now permits lasing action via the first excited state (upper spectrum of Fig. 7). For the range of cavity lengths studied (1-10 $\mathrm{mm}$ ) lasing on the ground state transition is never observed in the present single QD layer device. A similar dependence of lasing transition on cavity length has been reported in Ref. [23]. The inset to Fig. 7 shows the relative oscillator strengths of the lowest three transitions where their spontaneous emission intensities are plotted as a function of current. These intensities are measured from

\footnotetext{
$\| \Gamma_{\mathrm{ef}}=\left[I(0) / \sum_{i=\alpha}^{i=+\infty} I(i D)\right]$ where $I(x)$ is the transverse optical intensity profile.
} 


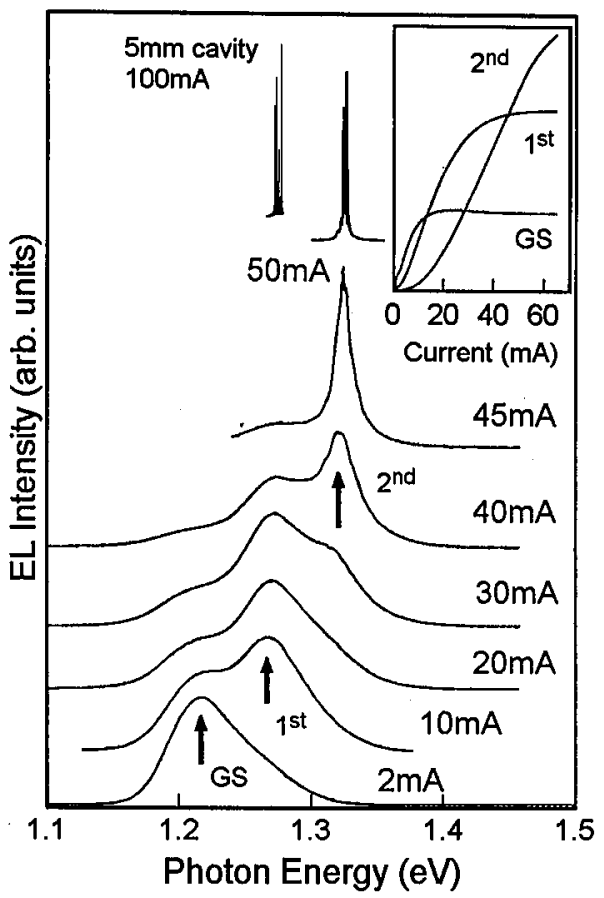

Fig. 7. Emission spectra of a $2 \mathrm{~mm}$ cavity laser device showing lasing occurring on the second excited state transition. The uppermost spectrum shows lasing for a $5 \mathrm{~mm}$ cavity device occurring on the first excited state transition. The inset shows the evolution with current of the spontaneous emission from the QD transitions.

a series of small windows formed in the top metal contacts of the laser device. With increasing current the transition intensities saturate as a result of state filling. Higher transitions exhibit a higher saturated intensity, consistent with a higher maximum gain. The relative maximum gains determined for the three transitions from the inset to Fig. 7 are found to be consistent with an internal loss of $10 \mathrm{~cm}^{-1}$ and the observation of lasing on the second and first excited states for 2 and $5 \mathrm{~mm}$ long cavities, respectively [26].

This section demonstrates how the photocurrent technique provides a relatively simple method to determine the modal gain of a quantum dot laser. Unlike the technique used in Ref. [20], which requires the measurement of a large number of laser devices, the present technique requires the measurement of a single, more easily fabricated mesa device. In addition it is a spectroscopic technique, allowing the spectral form of the gain to be determined.

\section{Conclusions}

Photocurrent spectroscopy has been shown to be a versatile and accurate technique for studying many important electronic properties of InAs/GaAs quantum dot structure and devices. Further advances in the field are likely to result from studies on dot ensembles with narrower linewidth, allowing for example better 
resolved excited state spectra to be obtained, and by the extension of photocurrent techniques to the study of single dots.

\section{References}

[1] D. Bimberg, M. Grundmann, N.N. Ledentsov, Quantum Dot Heterostructures, J. Wiley, Chichester 1999.

[2] J.J. Finley, M. Skalitz, M. Arzberger, A. Zrenner, G. Böhm, G. Abstreiter, Appl. Phys. Lett. 73, 2618 (1998).

[3] R.J. Warburton, C.S. Durr, K. Karrai, J.P. Kotthaus, G. Medeiros-Ribeiro, P.M. Petroff, Phys. Rev. Lett. 79, 5282 (1997).

[4] M. Grundmann, J. Christen, N.N. Ledentsov, J. Böhrer, D. Bimberg, S.S. Ruvimov, P. Werner, U. Richter, U. Gösele, J. Heydenreich, V.M. Ustinov, A.Yu. Egorov, A.E. Zhukov, P.S. Kop'ev, Zh.I. Alferov, Phys. Rev. Lett. 74, 4043 (1995).

[5] R.T. Collins, K. v. Klitzing, K. Ploog, Phys. Rev. B 33, 4378 (1986).

[6] L. Chu, M. Arzberger, A. Zrenner, G. Bohm, G. Abstreiter, Appl. Phys. Lett. 75, 2247 (1999).

[7] P.W. Fry, I.E. Itskevich, D.J. Mowbray, M.S. Skolnick, J.J. Finley, J.A. Barker, E.P. O'Reilly, L.R. Wilson, I.A. Larkin, P.A. Maksym, M. Hopkinson, M. Al-Khafaji; J.P.R. David, A.G. Cullis, G. Hill, J.C. Clark, Phys. Rev. Lett. 84, 334 (2000).

[8] M. Grundmann, O. Stier, D. Bimberg, Phys. Rev, B 52, 11969 (1995).

[9] M. Cusack, P.R. Briddon, M. Jaros, Phys. Rev. B 54, R2300 (1996); C. Pryor, Phys. Rev. B 57, 7190 (1998). This reference ( 8 band $k \cdot p$ theory) provides a summary of most earlier theoretical work.

[10] J. Kim, L.-W. Wang, A. Zunger, Phys. Rev. B 57, R9408 (1998).

[11] J.A. Barker, E.P. O'Reilly, unpublished.

[12] G. Bastard, Wave Mechanics Applied to Semiconductor Heterostructures, Halsted Press, New York 1988, p. 248.

[13] O. Stier, M. Grundmann, D. Bimberg, Phys. Rev. B 59, 5688 (1999).

[14] A.J. Williamson, L.W. Wang, A. Zunger, unpublished.

[15] W. Yang, H. Lee, P. Sercel, A. Norman, SPIE Photonics 1, 3325 (1999).

[16] R. Heitz, M. Veit, N.N. Ledentsov, A. Hoffmann, D. Bimberg, V.M. Ustinov, P.S. Kop'ev, Zh.I. Alferov, Phys. Rev. B 56, 10435 (1997).

[17] P.D. Buckle, P. Dawson, S.A. Hall, X. Chen, M.J. Steer, D.J. Mowbray, M.S. Skolnick, M. Hopkinson, J. Appl. Phys. 86, 2555 (1999).

[18] M.C. Bodefeld, R.J. Warburton, K. Karrai, J.P. Kotthaus, G. Medeiros-Ribeiro, P.M. Petroff, Appl. Phys. Lett. 74, 1839 (1999).

[19] A.R. Kovsh, A.E. Zhukov, D.A. Livshits, A.Yu. Egorov, V.M. Ustinov, M.V. Maximov, Yu.G. Musikhin, N.N. Ledentsov, P.S. Kop'ev, Zh.I. Alferov, D. Bimberg, Electron. Letts. 35, 1161 (1999); X.D. Huang, A. Stintz, C.P. Hains, G.T. Liu, J. Cheng, K.J. Malloy, IEEE Photonics Technol. Letts. 12, 227 (2000). 
[20] A.E. Zhukov, A.R. Kovsh, V.M. Ustinov, A.Yu. Egorov, N.N. Ledentsov, A.F. Tsatsul'nikov, M.V. Maximov, Yu.M. Shernyakov, V.I. Kopchatov, A.V. Lunev, P.S. Kop'ev, D. Bimberg, Zh.I. Alferov, Semicond. Sci. Technol. 14, 118 (1999); N. Kirstaedter, O.G. Schmidt, N.N. Ledentsov, D. Bimberg, V.M. Ustinov, A.Yu. Egorov, A.E. Zhukov, M.V. Maximov, P.S. Kop'ev, Zh.I. Alferov, Appl. Phys. Lett. 69, 1226 (1996).

[21] L.A. Coldren, S.W. Corzine, Diode Lasers and Photonic Integrated Circuits, J. Wiley, Chichester 1995.

[22] M. Grundmann, D. Bimberg, Jpn. J. Appl. Phys. 36, 4181 (1997).

[23] H. Shoji, Y. Nakata, K. Mukai, Y. Sugiyama, M. Sugawara, N. Yokoyama, H. Ishikawa, IEEE J. Sel. Top. Quantum Electron. 3, 188 (1997).

[24] S.Y. Hu, D.B. Young, S.W. Corzine, A.C. Gossard, L.A. Coldren, J. Appl. Phys. 76, 3932 (1994).

[25] M. Asada, Y. Miyamoto, Y. Suematsu, IEEE J. Quant. Electron. 22, 1915 (1986).

[26] P.W. Fry, L. Harris, S.R. Parnell, J.J. Finley, A.D. Ashmore, D.J. Mowbray, M.S. Skolnick, M. Hopkinson, G. Hill, J.C. Clark, J. Appl. Phys. 87, 615 (2000). 